
$\angle$ Research Square
Preprints are preliminary reports that have not undergone peer review.
They should not be considered conclusive, used to inform clinical practice, or referenced by the media as validated information.

\title{
Bioinformatics Analyses of Serine Acetyltransferase (SAT) Gene Family in Rice (Oryza sativa) and their Expressions under Salt Stress
}

\author{
Firat Kurt \\ Mus Alparslan Universitesi \\ Ertugrul Filiz ( $\nabla$ ertugrulfiliz@gmail.com ) \\ Duzce uni. https://orcid.org/0000-0001-9636-6389 \\ Adnan Aydın \\ Iğdır Üniversites: Igdir Universitesi
}

\section{Research Article}

Keywords: Sulfur, Rice, Hormone, Salt, Stress

Posted Date: April 26th, 2021

DOI: https://doi.org/10.21203/rs.3.rs-412789/v1

License: (a) (1) This work is licensed under a Creative Commons Attribution 4.0 International License. Read Full License

Version of Record: A version of this preprint was published at Molecular Biology Reports on August 13th, 2021. See the published version at https://doi.org/10.1007/s11033-021-06620-6. 


\section{Abstract}

Assimilation of sulfur to cysteine occurs in the presence of serine acetyltransferase (SAT). In this study, SAT genes in rice (Oryza sativa) were identified and analyzed using bioinformatics approaches. Also, these genes were tested under salt stress. OsSATs have two common motifs, bacterial transferase hexapeptide and acetyltransferase and underwent purifying selection. They have more similar protein sequences compared to Arabidopsis. However, there is structural and functional divergence among OsSATs which may be driven by the segmental and tandem duplications. Purifying selection and gene duplications may also have effect leading to variation of specificity and selectivity of OsSATs. In this regard, Asp (D), His (H), Gly (G), Thr (T), Arg (R), Ala (A), and Leu (L) are identified as well-conserved residues in their active sites which have an indicator role on their functions. The OsSATs expressions in different tissues, organs and under hormones showed that jasmonic acid was main hormone inducing the expressions of OsSAT1;1, OsSAT2;1, and OsSAT2;2 whereas auxin and abscisic acid only triggered OsSAT1;1 expression. On the other hand, wet-lab expressions of OsSATs in this study indicated that OsSAT1;1, OsSAT1;2 and OSSAT1;3 genes were upregulated under different exposure times of salt stress. OsSAT1;1 is the only OsSAT induced by various situmuli. The findings can be used by plant breeders and genetic engineers to develop new rice varieties having optimal growth and stress tolerance.

\section{Introduction}

Sulfur (S) is a vital macronutrient for plants and involved in many biochemical reactions. S is the fourth most important plant nutrient after $\mathrm{N}$, $\mathrm{P}$, and $\mathrm{K}$. It is found in amino acids (cysteine and methionine), vitamins and cofactors, glutathione and phytochelatins [1]. Plants can use the reduced sulfate ( $\mathrm{SO}_{4}{ }^{2-}$ ) and convert it to sulfide $\left(\mathrm{S}^{2-}\right)$. Sulfide is used to integrate $S$ into cysteine (Cys) in the cytosol. Cys biosynthesis is a basic process either as a precursor or donor of key S compounds in plants. While Cys synthesis is observed in the cytosol, plastids, and mitochondria, the sulfate reduction pathway is localized in plastids of plants [2, 3]. The Cys synthesis takes place in the presence of two enzymes: serine acetyltransferase (SAT or SERAT) and 0acetylserine(thiol)lyase (OASTL). The former leads to synthesizing intermediary product O-acetylserine (OAS) from acetyl-CoA and serine. The latter uses the sulfide and OAS for production of Cys as a cofactor in the presence of pyridoxal-5'-phosphate. These two enzymes are called hetero-oligomeric cysteine synthase complex (CSC) [4-6].

The ancestral SAT gene was a host origin gene and not evolved from the cyanobacterial endosymbiont [7]. A total of five serine acetyltransferases (SAT or SERAT; EC 2.3.1.30) were identified in Arabidopsis thaliana [8]. AtSAT1, AtSAT3, and AtSAT5 are found to localized in plastid, mitochondria, and cytoplasm, respectively [2]. Additionally, mitochondrion is identified as the most important compartment for 0-acetylserine (OAS) synthesis in Arabidopsis, which is the precursor of Cys [3]. Similarly, cell-distribution of SAT activities in pea (Pisum sativum) leaves is found at the highest level in mitochondria followed by chloroplast and cytoplasm [2]. The other two AtSATs, AtSAT2 and AtSAT4, are localized in cytoplasm but their protein sequences show variations from other SATs and are expressed at low level [9]. The overexpressed of AtSAT1 in maize does not cause any negative effect on Arabidopsis's growth and it enhances 10-kDa y-zein storage protein during endosperm development [10].

In this study, rice (Oryza sativa) SAT (OsSATS) genes/proteins were identified at genome-wide scale, then bioinformatics analyses were performed (sequence, motif, phylogenetic, expression, and protein modeling) to find out structural and functional divergences of SATs in rice. Also, the responses of OSSAT genes were tested to offer more insights about their regulations under salt stress.

\section{Materials And Methods}

\section{Identification of $S A T$ genes and sequence feature analysis}

Firstly, Q42588 AtSAT1 (At1g55920), Q8S895 AtSAT2 (At2G17640), Q39218 AtSAT3 (At3g13110), Q8W2B8 AtSAT4 (At4g35640), and Q42538 AtSAT5 (At5g56760) sequences in Arabidopsis were retrieved from UniProt database (https://www.uniprot.org/). AtSATs were only used for comparative analyses in this study. These protein sequences were searched against rice genome (Oryza sativa v7_JGI) on Phytozome database v12 (https://phytozome.jgi.doe.gov/pz/portal.html\#!info?alias=Org_Osativa) with an E-value < 1E-20 [11]. Later, the presence of PF06426 (SATase_N) domain structure was validated using Pfam database v32 (https://pfam.xfam.org/) [12]. Genomic and coding sequences corresponding to SATs were collected from Phytozome database for further bioinformatics analyses. Physicochemical properties of SAT proteins such as length, molecular weight, and isoelectric point ( $p l$ values were obtained from ProtParam tool (web.expasy.org/protparam) [13]. The sequence identity matrix for Arabidopsis and rice SATs were generated using Bioedit v7.2.5 [14]. Annotations of SAT proteins were analyzed using PANNZER (Protein ANNotation with Z-scoRE) server (http://ekhidna2.biocenter.helsinki.fi/sanspanz/) [15]. The conserved motif analysis was performed using MEME suit (motif-based sequence analysis tools) (http://meme-suite.org/). Max motif number and min-max motif width parameters on the platform were set to, in turn, 10 and 6-50 [16].

\section{Nucleotide and phylogenetic analyses}

Maximum likelihood estimate of transition/transversion bias (R) was estimated under the Kimura [17] using two-parameter model. The estimate of transition/transversion bias and G+C content were calculated using DnaSP v6.12.01 [18]. Molecular phylogenetic analysis was performed according to the maximum likelihood (ML) method using JTT matrix-based model [19]. Initial tree(s) for the heuristic search were obtained automatically by applying Neighbor-Join and BioNJ algorithms to a matrix of pairwise distances, which are estimated using a JTT model, and then topology with superior log likelihood value was selected. The analysis involved 11 amino acid sequences with 266 positions after all positions containing gaps and missing data were eliminated. Evolutionary analyses were conducted in MEGA7 [20]. 


\section{Selection, gene duplication and synteny analyses}

Nucleotide sequences of rice SAT genes were analyzed using DnaSP v6.12.01 [18], in terms of polymorphic sites, DNA polymorphism, genetic variation level ( $\pi$ and $\theta$ ), and Tajima's D [21]. Ka and Ks values were calculated for duplicated gene pairs using DnaSP v6.12.01 [18]. Gene duplications analyses were conducted according to two following criteria: If the alignment of the coding nucleotide sequences covers $70 \%$ of the longest genes and the amino acid identity between the sequences are $\geq 70 \%$, gene of interest is assumed to be duplicated [22].

Synteny analyses were conducted on Circoletto platform [23]. With this aim, processed genes of Arabidopsis, Brachypodium distachyon, Solanum lycopersicum, and Zea mays were downloaded from Plant Genome Duplication Database [24]. Then the local blast database was constructed in Bioedit [14] and the blast results were fed to Circoletto.

\section{Digital expression and co-expression analyses}

Digital expression profiles of OSSAT genes were obtained from Rice Expression Profile Database (RiceXPro) v3 (http://ricexpro.dna.affrc.go.jp/) (Sato et al. 2013a). Co-expression network was constructed using RiceFREND co-expression database. RiceFRIEND contains expression profiles obtained from microarray analyses of tissues/organs of rice plant under natural field conditions and various treatments (http://ricefrend.dna.affrc.go.jp/) (Sato et al. 2013b)

\section{Analyses of secondary and tertiary structures, predicted binding sites, and protein interactions}

Secondary structure analyses of OsSAT proteins were conducted using SOPMA server (https://npsa-prabi.ibcp.fr/cgi-bin/npsa_automat.pl? page=/NPSA/npsa_sopma.html) [27]. Tertiary models were predicted by using Phyre ${ }^{2}$ server (Protein Homology/analogY Recognition Engine V 2.0 (https://www.sbg.bio.ic.ac.uk/phyre2/) [28]. The predicted 3D models were visualized using Swiss-PdbViewer [29]. The independent comparisons of biomolecular 3D structures were performed using CLICK server (http://www.mspc.bii.a-star.edu.sg/click) [30]. The binding sites of OsSAT protein sequences were identified using InterProScan tool (http://www.ebi.ac.uk/interpro/search/sequence-search) [31]. Protein-protein interaction network was constructed using STRING database v11 (https://string-db.org/) [32].

\section{Plant material and growth conditions}

Salt tolerant local Karacadag variety was used as plant material. Rice seeds were sown in 10 -cm-square pots containing $40 \%$ perlite and $60 \%$ peat. The plants were grown in the growth cabinet with following cycle: an average of $50 \%$ humidity at $25^{\circ} \mathrm{C}$ at 8 -hour dark stage and $30{ }^{\circ} \mathrm{C}$ at 16 -hour bright stage. 4-5 plants were grown for each experimental group. Third true leaves of rice plants were exposed to $200 \mathrm{mM} \mathrm{NaCl}$ and leaf samples were collected at the $3^{\text {rd }}, 12^{\text {th }}$ - and $24^{\text {th }}$ - hour. Leaf samples were immediately stored in RNA/eater stabilization solution (Invitrogen, Cat No: AM7021).

\section{RNA isolation and gene expression analysis}

RNA isolation was conducted by TRIzol reagent method (Ambion, Ref: 15596026). The quality and quantity of the isolated RNA were measured using $2 \%$ agarose gel and nano-drop spectrophotometer (Maestrogen, MN-913). A total of 12 samples (i.e. 3 biological replicates from each sample) were used in Real-Time qPCR studies. Genomic DNA elimination were carried out with these 12 samples by taking into account the protocol of the DNasel kit (Cat: EN0521). RNAs were then converted to cDNA according to the kit's protocol. qPCR studies were performed with the QIAGEN Rotor-Gene Q 5-Plex instrument (Bustin et al., 2009). The OsSATs were expressed using the AMPIGENE qPCR Green Mix (Cat No: ENZ-NUC104-10000) commercial kit. OsActin/l gene, identified for salt stress in rice, is used as reference gene [33].

Relative Quantification $2^{-\Delta \Delta C}$ (Livak and Schmittgen 2001) method was used to compare gene expression. Gene expression data were analyzed with Rotor-Gene Q 2.3.5 software. Primers used in the study (Table 1) were designed using Primer3. v. 0.4.0 program (Untergasser et al. 2012)

(http://bioinfo.ut.ee/primer3-0.4.0/) and synthesized by Macrogen Crop. (Amsterdam, The Netherlands).

Table 1

Primers used for RT-qPCR analysis of SATs genes. 


\begin{tabular}{|c|c|c|}
\hline Primer ID & Sequences $\left(5^{\prime}->3^{\prime}\right)$ & Gene Name \\
\hline \multirow[t]{2}{*}{ OsSAT1;1 } & F:GACGAGTCGTGGGTCTGG & \multirow[t]{2}{*}{ LOC_Os01g52260.1 CDS } \\
\hline & R:GGTGGCGTAGAGGAACGAC & \\
\hline \multirow[t]{2}{*}{ OsSAT1;2 } & F:ACTCCAGCACGAGCATGAC & \multirow[t]{2}{*}{ LOC_Os02g10830.1 CDS } \\
\hline & R:CCTTGATCTGGGACCACAC & \\
\hline \multirow[t]{2}{*}{ OsSAT3 } & F:TGAAACTGCCATTGTTGGAA & \multirow[t]{2}{*}{ LOC_Os03g04140.1 CDS } \\
\hline & R:TGACCAATCTTGGGGTGTCT & \\
\hline \multirow[t]{2}{*}{ OsSAT2;1 } & F:ATCGTGCTACGGGACGTG & \multirow[t]{2}{*}{ LOC_Os03g08660.1 CDS } \\
\hline & R:GTTACCCCGGGAAGCTCT & \\
\hline \multirow[t]{2}{*}{ OsSAT2;2 } & F:ACTACGTGTGGGACGTGCT & \multirow[t]{2}{*}{ LOC_Os03g10050.1 CDS } \\
\hline & R:ACAGGACGAGGTCGTGGTAG & \\
\hline \multirow[t]{2}{*}{ OsSAT1;3 } & F:ACAACGTCTCCATCCTCCAC & \multirow[t]{2}{*}{ LOC_Os05g45710.1 CDS } \\
\hline & R:CAGAACACCATCCCCAАTCT & \\
\hline
\end{tabular}

\section{Results}

\section{Genome-wide identification and sequence analyses of SATs}

A total of six non-redundant SAT genes were identified in rice genome using five AtSAT protein sequences as references: LOC_0s01g52260 (OsSAT1;1), LOC_Os02g10830 (OSSAT1;2), LOC_Os03g04140 (OsSAT3), LOC_Os03g08660 (OsSAT2;1), LOC_Os03g10050 (OsSAT2;2), and LOC_0s05g45710 (OsSAT1;3) (Table 2). The protein lengths of SATs are ranged from 298 to 391 amino acid residues; molecular weights were found between 30.44 and $42.72 \mathrm{kDa}$. All SATs are predicted to be acidic character ( $p / \leq 7)$ except for AtSAT3. Exon numbers range from one to 10 . AtSAT2 and 4 (SAT3 family) and OsSAT3 have 10 exons. All SAT proteins in the study contain the serine acetyltransferase N-terminal domain structure (SATase_N, PF06426). In addition, bacterial transferase hexapeptide (PF00132) domain was identified as one or two repeats.

Table 2

Properties of SAT members in Arabidopsis and rice.

\begin{tabular}{|c|c|c|c|c|c|c|c|}
\hline Phytozome ID & Species & $\begin{array}{l}\text { Exon } \\
\text { no }\end{array}$ & $\begin{array}{l}\text { Protein } \\
\text { length } \\
\text { (aa) }\end{array}$ & $\begin{array}{l}\text { Domain } \\
\text { family }\end{array}$ & Repeat number & $\begin{array}{l}\text { Mol. wt. } \\
\text { (kDa) }\end{array}$ & pl \\
\hline At1g55920 (SAT1, SERAT2;1) & A. thaliana & 1 & 314 & PF06426 & 2 & 34.25 & 6.45 \\
\hline At2g17640 (SAT2, SERAT3;1) & A. thaliana & 10 & 323 & PF06426 & 2 & 34.53 & 5.81 \\
\hline At3g13110 (SAT3, SERAT2;2) & A. thaliana & 1 & 391 & PF06426 & 1 & 42.72 & 7.73 \\
\hline At4g35640 (SAT4, SERAT3;2) & A. thaliana & 10 & 355 & PF06426 & 1 & 38.42 & 5.56 \\
\hline At5g56760 (SAT5, SERAT1;1) & A. thaliana & 2 & 312 & PF06426 & 2 & 32.77 & 6.70 \\
\hline LOC_Os01g52260 (OsSAT;1) & O. sativa & 1 & 303 & PF06426 & 2 & 31.85 & 6.66 \\
\hline LOC_Os02g10830 (OSSAT1;2) & O. sativa & 1 & 298 & PF06426 & 2 & 30.44 & 5.33 \\
\hline LOC_Os03g04140 (OsSAT3) & O. sativa & 10 & 354 & PF06426 & 1 & 37.52 & 6.37 \\
\hline LOC_Os03g08660 (OsSAT2;1) & O. sativa & 1 & 301 & PF06426 & 1 & 31.50 & 5.91 \\
\hline LOC_Os03g10050 (OsSAT2;2) & O. sativa & 1 & 315 & PF06426 & 1 & 32.92 & 6.20 \\
\hline LOC_Os05g45710 (OsSAT1;3) & O. sativa & 1 & 314 & PF06426 & 1 & 33.09 & 6.38 \\
\hline
\end{tabular}

To provide more insight about protein sequence diversity of SATs, sequence identity matrix was constructed (Table 3). The lowest identity value was found 0.325 between AtSAT2 and AtSAT3 whilst the highest value was found between OsSAT2;1 and OsSAT2;2 as 0.914. The mean identity value of SAT proteins was 0.476 . 
Sequence identity matrix of SATs in Arabidopsis and rice

\begin{tabular}{|c|c|c|c|c|c|c|c|c|c|c|c|}
\hline & AtSAT1 & AtSAT2 & AtSAT3 & AtSAT4 & AtSAT5 & Os1 & Os2 & Os3 & Os4 & Os5 & Os6 \\
\hline AtSAT1 & ID & & & & & & & & & & \\
\hline AtSAT2 & 0.390 & ID & & & & & & & & & \\
\hline AtSAT3 & 0.611 & 0.325 & ID & & & & & & & & \\
\hline AtSAT4 & 0.393 & 0.700 & 0.341 & ID & & & & & & & \\
\hline AtSAT5 & 0.495 & 0.425 & 0.409 & 0.407 & ID & & & & & & \\
\hline Os1 & 0.503 & 0.417 & 0.426 & 0.422 & 0.685 & ID & & & & & \\
\hline Os2 & 0.477 & 0.398 & 0.393 & 0.393 & 0.609 & 0.741 & ID & & & & \\
\hline Os3 & 0.386 & & & 0.563 & 0.408 & 0.405 & 0.400 & ID & & & \\
\hline Os4 & 0.473 & 0.367 & 0.383 & 0.356 & 0.444 & 0.506 & 0.498 & 0.356 & ID & & \\
\hline Os5 & 0.496 & 0.374 & 0.406 & 0.369 & 0.476 & 0.523 & 0.507 & 0.372 & 0.914 & ID & \\
\hline Os6 & 0.498 & 0.398 & 0.407 & 0.407 & 0.703 & 0.818 & 0.700 & 0.400 & 0.481 & 0.501 & ID \\
\hline
\end{tabular}

\section{Conserved motif analysis}

Regarding conserved motifs, 10 conserved motifs were detected (Table 4 and Fig. 1). The motif 3 was found to be related with PF00132 (Bacterial transferase hexapeptide). Motif 4 and 5 were related with PF06426 (serine acetyltransferase) domain structure. The motif 1, 2, 3, 4, and 5 were present in all SATs whereas other motifs were found in different numbers.

Table 4

The details of conserved motif sequences in SAT proteins

\begin{tabular}{|c|c|c|c|c|}
\hline No. & Motif sequence & S & W & Domain \\
\hline 1 & IGKGILLDHATGVVIGETAVVGBNVSILHGVTLGGTGKESGDRHPKIGDG & 11 & 50 & NF \\
\hline 2 & YSHCLLNYKGFLALQAHRVAHKLWAQGRKALALALQSRVSEVFAVDIHPA & 11 & 50 & NF \\
\hline 3 & VLIGAGATILGNVKIGAGAKIGAGSVVLKDVPPRTTAVGNPARLIGGKDE & 11 & 50 & PF00132 \\
\hline 4 & WDQIKAEAKRDAEKEPILSSFLYASVLSHPSLERALAFHLANKLCNPTLL & 11 & 50 & PF06426 \\
\hline 5 & FAGVLAAHPEJRAAVRADLLAAKDRDPAC & 11 & 29 & PF06426 \\
\hline 6 & IPGESMDHTSFISEWSDYTI & 6 & 20 & NF \\
\hline 7 & QDPSLTMKHDATREFFQHVAVAYKDDKPN & 3 & 29 & NF \\
\hline 8 & MAACIDKWPTGKPQ & 4 & 14 & NF \\
\hline 9 & RLPEKFYCVLPDCTATDRPV & 2 & 20 & NF \\
\hline 10 & TQLYDL & 11 & 6 & NF \\
\hline
\end{tabular}

\section{Nucleotide and phylogenetic analyses}

$\mathrm{R}$ value, the ratio of transition to transversion, were estimated to provide insight into DNA sequence evolution and phylogeny reconstruction. Also, $\mathrm{G}+\mathrm{C}$ contents of OsSATs were calculated to predict probable functional variations of the genes which are important for organisms in gaining adaptation to its environment. The estimated transition/transversion bias (R) was found as 0.71 , indicating genetic variations. $G+C$ contents were found $71.82 \%, 75.25 \%$, $51.92 \%, 72.63 \%, 72.47 \%$, and $68.68 \%$ for OsSAT1;1, OSSAT1;2, OsSAT3, OsSAT2; 1 , OsSAT2;2, and OsSAT1;3, respectively.

Phylogenetic analysis indicated that AtSATs and OsSATs split into two major clades (Group A and B). Group A also divided into two subclades (Fig. 2). OsSAT2;1 (LOC_Os03g08660) and OsSAT2;2 (LOC_Os03g10050) clustered with AtSAT1 (SAT2;1) and AtSAT3 (SAT2;1) in subclade A1, whereas OsSAT1;3 (LOC_Os05g45710), OsSAT1;1 (LOC_Os01g52260), and OsSAT1;2 (LOC_Os02g10830) grouped with AtSAT5 (SAT1;1) in subgroup A2. OsSAT3 
(LOC_Os03g04140) gene clustered with AtSAT2 (SAT3;1) and AtSAT4 (SAT3;2) and separated from rest of SATs in group B (100\%), indicating genetic divergence. This separation is related to OsSAT3 having the highest identity scores, in turn, with AtSAT4 and AtSAT2.

\section{Selection, gene duplication and synteny analyses}

Nucleotide variations of six OsSAT genes were analyzed using two selection analyses: Tajima's D and Ka/Ks tests. In Tajima's D test, the number of polymorphic (segregating) sites in OsSAT genes were identified as 516 , of which $47.1 \%(243 / 516)$ were singleton variable sites and $30.4 \%$ (273/516) were parsimony informative sites. 306 sites were invariable (monomorphic) and nucleotide diversity was found 0.33 and 0.27 for $\pi$ and $\theta$ parameters, respectively. Tajima's D was found 1.22, indicating purifying (negative) selection.

In the second selection test, the nonsynonymous $(K a)$ and synonymous $(K s)$ substitution rates between the duplicated gene pairs were calculated. Ka/Ks values for all OSSAT genes were found less than one, validating previous finding that OsSAT genes were subjected to purifying selection. Moreover, gene duplications analyses indicated that three segmental duplications and one tandem duplication occurred, suggesting that these duplications are the major force for SAT gene expansion (Table 5).

Table 5

Segmental and tandem duplications of SAT paralogous pairs in rice genome

\begin{tabular}{|c|c|c|c|c|c|}
\hline SAT group & Chr. location & Duplication type & $\mathrm{Ka}$ & Ks & $\mathrm{Ka} / \mathrm{Ks}$ \\
\hline \multirow[t]{2}{*}{ OsSAT1;1 OsSAT1;2 } & Chr1 & Segmental & 0.114 & 0.184 & 0.619 \\
\hline & Chr2 & & & & \\
\hline \multirow[t]{2}{*}{ OsSAT1;1 OsSAT1,3 } & Chr1 & Segmental & 0.073 & 0.296 & 0.247 \\
\hline & Chr5 & & & & \\
\hline \multirow[t]{2}{*}{ OsSAT1;2 OsSAT1;3 } & Chr2 & Segmental & 0.146 & 0.363 & 0.402 \\
\hline & Chr5 & & & & \\
\hline \multirow[t]{2}{*}{ OsSAT2;1 OsSAT2;2 } & Chr3 & Tandem & 0.018 & 0.023 & 0.783 \\
\hline & Chr3 & & & & \\
\hline
\end{tabular}

To understand gene duplication dynamics of OSSAT genes four comparative syntenic maps of rice, associated with four representative species, were generated. (Figure 3). Six OSSAT genes had a syntenic relationship with five genes in Arabidopsis, followed by four genes in B. distachyon, four genes in tomato, and three genes in maize.

\section{Distinct expression profiles of OSSAT genes}

To better understand the functions of OSSAT genes in the cell metabolism, gene expression levels in different tissues and organs were displayed as a heatmap in Fig 4. For digital expression analysis, five OsSAT genes were obtained from Rice Expression Profile Database (RiceXPro) except for OsSAT1;2 (LOC_Os02g10830) gene. In general, OsSAT genes in stem, inflorescence, anther, pistil, lemma, palea, and embryo tissues/organ were expressed at low level. Particularly, OsSAT2;1 and OsSAT2;2 genes were down-regulated as about -3-fold change. Also, these two genes were downregulated in inflorescence and anther tissues. In leaf blade and sheath, OsSAT genes commonly were upregulated. OsSAT2;1 and OsSAT2;2 genes were upregulated about three-fold changes in leaf blade.

In the second step, gene expression profiles of OSSAT genes were evaluated at five different time points depending on six types of plant hormone applications (abscisic acid, gibberellin, auxin, brassinosteroid, cytokinin, and jasmonic acid) (Fig. 5). Jasmonic acid treatment especially causes upregulation of OSSAT genes in terms of gene expression levels. Particularly OsSAT1;1 gene was upregulated nearly four-fold by jasmonic acid. Also, it was found that the optimal time point for the expression to increase was one hour for OsSAT1;1 gene under jasmonic acid treatment. Also, OsSAT1;1 responded to the auxin and abscisic acid treatments at the third hour of exposure despite its responses were not as high as to that of jasmonic acid. The up-regulation of OsSAT1; 1 gene also was observed under abscisic acid and auxin treatments. OsSAT2;1 and OsSAT2;2 genes were upregulated at the first hour of exposure to jasmonic acid. Overall, levels of gene expressions under hormone treatments supported that OsSAT1;1, OsSAT2;1, and OsSAT2;2 genes were positively regulated by hormone treatments.

\section{Co-expression analysis of OsSAT genes}

The co-expression network of OSSAT genes was constructed using RiceFREND database. Co-expression network of OsSAT genes displayed that Os04g0577500, Os11g0524300, Os06g0167400, Os06g0690700, Os12g0641300, Os04g0488700, and Os07g0589000 were seven first neighbor genes

Page 6/17 
that co-expressed with OsSATs (Fig. 6). Os04g0488700 (similar to PHY3, AGC kinase) was co-expressed with OsSAT1;1 gene. The AGC kinase family is one of seven kinase families and they are conserved in all eukaryotic genomes. AGC kinases in plants play roles in modulation of kinase activity by external stimuli [34]. Os12g0641300 (similar to Zn-dependent hydrolases of the beta-lactamase fold) was identified as a co-expressed gene with OsSAT1;3. Os07g0589000 (lateral organ boundaries, LOB domain containing protein) was co-expressed with OsSAT2;1 and OsSAT2;2. Lateral organ boundaries domain (LBD) proteins contain lateral organ boundaries (LOB) domain that are key regulators for plant organ development such as photomorphogenesis, plant regeneration, and pollen development [35]. OsSAT3 was co-expressed with Os04g0577500 (TatD-related deoxyribonuclease family protein), Os11g0524300 (protein of unknown function DUF1001 family protein), Os06g0167400 (di-trans-poly-cis-decaprenylcistransferase family protein), and Os06g0690700 (similar to potential cadmium/zinc-transporting ATPase HMA1). TatD is conserved protein found in all living organisms and participates in DNA fragmentation during apoptosis in eukaryotic cells [36]. Heavy metal pumps (P1B-ATPases) are vital for cellular heavy metal homeostasis.

Arabidopsis thaliana contains eight P1B-ATPase genes (heavy metal ATPases 1-8 (HMA1-HMA8) members [37].

\section{Annotations of SAT proteins}

The gene ontology (GO) analyses of OsSAT proteins were performed using PANNZER server, in terms of biological process, molecular function, and cellular component (Fig. 7). Sulfur amino acid biosynthetic process (G0:0000097), L-serine metabolic process (G0:0006563), biosynthetic process from serine (GO:0006535), cellular amino acid biosynthetic process (GO:0008652), sulfate assimilation (G0:0000103), and response to sulfate starvation (G0:0009970) were identified as biological processes in which OsSATs are involved (Fig. 7A). Serine 0-acetyltransferase activity (G0:0009001), terpene synthase activity (G0:0010333), magnesium ion binding (GO:0000287), zinc ion binding (G0:0008270), and protein binding (G0:0005515) were predicted molecular functions which are OsSATs are carried out (Fig. 7B). In addition, cytoplasm (G0:0005737), intrinsic component of membrane (G0:0031224), intracellular part (GO:0044424), and membrane-bounded organelle (G0:0043227) were identified as cellular components in which OsSATs function (Fig. 7C). When the results of GO analyses were evaluated, it was clearly observed that amino acid synthesis is the most prominent biological process for OsSATs.

\section{Secondary and tertiary structure analyses of OsSATs}

According to the secondary structure analyses, there are structural variations among OsSAT proteins. The alpha helix, extended strand, beta turn, and random coil percentages (\%) were found between $36.94-42.62,17.46-21.19,7.38-10.45$, and $28.25-34.65$, respectively (Supp. Table 1). The predicted 3D structures of OsSAT proteins (Fig. 8) were found reliable due to and their Ramachandran values ranging from $96 \%$ to $99 \%$ in core and allowed regions. Homologous proteins from different organisms can be recognized using sequence comparison because amino acid substitutions in particular positions are prevented by strong selective constraints [38] . These structural variations at secondary and tertiary levels may be associated with SAT proteins' functional flexibilities.

The 3D structural similarities (\%) were identified using six rice, five Arabidopsis, and one soybean SAT proteins on CLICK structure comparison server (Table 6). In general, the 3D structural similarity values were identified between $68.36 \%$ and $88.45 \%$. The highest ( $88.45 \%$ ) and lowest (68.36\%) similarity values were found in turn between OsSAT1;1 \& AtSAT4 and OsSAT3 \& GmSAT. When Arabidopsis and rice similarity values were compared, the similarity was observed over $80 \%$, indicating well-conserved SAT protein structure. The protein having the lowest similarity values with other SATs was OsSAT3 protein, proving structural divergence among SATs [9].

Table 6

The 3D structure overlap (\%) of rice, Arabidopsis, and soybean SATs using CLICK structure comparison server

\begin{tabular}{|lllllll|}
\hline & Os1 & Os2 & Os3 & Os4 & Os5 & Os6 \\
\hline AtSAT1 & 82.84 & 87.58 & 79.30 & 85.71 & 82.80 & 79.94 \\
\hline AtSAT2 & 83.50 & 87.25 & 84.21 & 85.38 & 84.76 \\
\hline AtSAT3 & 87.79 & 88.26 & 73.45 & 86.05 & 86.03 & 87.85 \\
\hline AtSAT4 & 88.45 & 88.26 & 72.88 & 86.71 & 84.44 \\
\hline AtSAT5 & 79.87 & 82.21 & 84.62 & 80.73 & 79.17 & 77.14 \\
\hline GmSAT & 79.87 & 81.21 & 88.36 & 81.73 & 77.71 \\
\hline $\begin{array}{l}\text { Os1: LOC_Os01g52260 (OsSAT1;1), Os2: LOC_Os02g10830 (OsSAT1;2), Os3: LOC_Os03g04140 (OsSAT3), Os4: LOC_Os03g08660 (OsSAT2;1), Os5: } \\
\text { LOC_Os03g10050 (OsSAT2;2), Os6: LOC_Os05g45710 (OsSAT1;3), Gm: Glycine max (PDB code: 4N69) }\end{array}$ & & & \\
\hline
\end{tabular}

\section{Predicted active sites of OsSATs}

The identification of catalytic residues of enzymes is an indispensable step for understanding the functions of enzymes [39] . In this study, active site predictions of OsSATs were performed using InterPro 74.0 server (Table 7). Particularly, Asp (D), His (H), Gly (G), Thr (T), Arg (R), Ala (A), and Leu (L) residues were conserved at different positions in all OsSATs; in contrast, some residues such as 248M (Met), 249Q (GIn), and 292A (Ala) residues were only 
identified in LOC_Os03g04140 (OsSAT3) protein, suggesting functional divergence of SAT3 protein in rice. In general perspective, it was found that similar amino acid residues were present in the predicted active binding sites.

Table 7

Predicted active sites of OsSAT proteins

\begin{tabular}{|ll|}
\hline Protein name & Active sites \\
\hline $\begin{array}{l}\text { OsSAT1;1 } \\
\text { (LOC_0s01g52260) }\end{array}$ & 189D, 190H, 209L, 210H, 216G, 217T, 224R, 225H, 236A, 251K, 253G, 254A, 257L, 259L, 267T, 272P \\
\hline OsSAT1;2 (LOC_Os02g10830) & 182D, 183H, 202L, 203H, 209G, 210T, 217R, 218H, 229A, 244K, 246G, 247A, 250L, 252L, 260T, 265P \\
\hline LOC_Os03g04140 (OsSAT3) & 228D, 229H, 248M, 249Q, 255G, 256T, 263R, 264H, 275A, 290M, 292A, 293A, 296L, 298L, 306M, 311P \\
\hline OsSAT2;1 (LOC_Os03g08660) & 197D, 198H, 217L, 218H, 224G, 225T, 232R, 233H, 244A, 259E, 261G, 262A, 265I, 267L, 275T \\
\hline OsSAT2;2 (LOC_Os03g10050) & 203D, 204H, 223L, 224H, 230G, 231T, 238R, 239H, 250A, 265K, 267G, 268A, 271V, 273L, 281T, 286P \\
\hline OsSAT1;3 LOC_Os05g45710 & 195D, 196H, 215L, 216H, 222G, 223T, 230R, 231H, 242A, 257K, 259G, 260A, 263V, 265L, 273T, 278P \\
\hline
\end{tabular}

\section{The expression of OsSATs under salt stress}

In this study, OsSATs responses under 3, 12, 24-h salt treatments were investigated (Fig. 9). OsSAT2;1, OsSAT2;2 and OsSAT3 were downregulated under all exposure times. The magnitude of OSSAT2;1 expression to all salt treatments was the lowest compared to other OsSATs. On the other hand, OsSAT2;2 and OsSAT3 responded to exposure times in a similar way. The expressions of OsSAT1;1 and OsSAT1;2 and OsSAT1;3 increased depending on the 3, 12, and 24-hour $\mathrm{NaCl}$ treatments. OsSAT1;2 was generally expressed at the highest level under all exposure times. Lastly, the responses of OsSAT1;2 and OsSAT1;3 to salt exposure times were the highest at 24-h NaCl treatment. Overall, OsSAT1;1 and OSSAT1;2 and OsSAT1,3 are responsive genes to different salt exposure times; and $O S S A T 1 ; 2$ and $O S S A T 1,3$ were particularly upregulated by 24-hour salt treatment.

\section{Discussion}

Sequence, nucleotide and phylogenic analyses of Arabidopsis and rice proteins showed that there are sequential and phylogenetic divergences among SATs (Kawashima et al. 2005). AtSATs and OsSATs were not separated from each other completely in phylogenetic analysis due to having the same number of exons and relatively higher identity scores. For example, AtSAT2/4 and OsSAT3 genes have 10 exons with identity values above $50 \%$ unlike the rest of SATs. In terms of identity values of plants of interest, OsSATs have more similar (0.541) protein sequences compared to AtSATs (0.450). Nonetheless, the selection analyses of OSSAT genes showed that SATs are subjected to the purifying selection. Purifying selection, also known as background selection, reduces genetic diversity and shapes it in natural population [40]. Consequently, it may be suggested that the genetic diversity of OSSAT genes decreased as a result of purifying selection.

$\mathrm{R}$ value, the ratio of transition to transversion, were estimated for DNA sequence evolution and phylogeny reconstruction [41]. In any genome, transitions ( $T \leftrightarrow C$ and $A \leftrightarrow G$ ) are observed at higher frequencies than transversions ( $T \leftrightarrow A, T \leftrightarrow G, C \leftrightarrow A$, and $C \leftrightarrow G)$. In this study, the estimated transition/transversion bias (R) was found as 0.71 , indicating genetic variations. Proving this result, GC contents of OsSATs also showed a considerable variation. Genomic DNA base composition (GC content) affects genes' functions and adaptation of species to its environment and it may play roles in complex gene regulation [42]. Consequently, it can be concluded that the action of purifying selection may increase the specificity and the selectivity of SATs in rice metabolism, leading to variations of $\mathrm{G}+\mathrm{C}$ contents. In addition to that, we found that segmental and tandem duplications are driving force of OsSATs evolution. It is known that gene duplication is one of types of genomic change that can lead to evolutionary changes. The duplicated genes can contribute to the evolution of novel functions including adaptation to stress, induction of disease resistance, production of floral structures [43] and expansion of gene families [44]. As is found in this study, it is reported that SAT2;1/SAT2;2 and SAT3;1/SAT3;2 isoforms in Arabidopsis were also duplicated gene pairs [45].

Although all SATs contain serine acetyltransferase N-terminal domain structure (SATase_N, PF06426) and bacterial transferase hexapeptide (PF00132) as their common motifs, there is still variations in motif structures. The presence of protein motifs may play important roles in protein function. The motifs in active sites of proteins are well-conserved [46], suggesting that these variations in motif structures may be connected with functional diversities of SATs in plants.

Expression patters of OsSATs showed difference according to tissues and organs, and treatments. This result is validated by the co-expression maps of OsSAT genes showing their involvement in various metabolic pathways and their association with gene families with different functions. When the expression levels of OsSATs by organs are taken into consideration, it was observed that the expression levels of OsSAT2;1 and OsSAT2;2 genes showed more dynamic profiles. Jasmonic acid is the only hormone activating expression of OsSAT1;1, OsSAT2;1, and OsSAT2;2. Besides, OsSAT1;1 was the only OsSAT induced by auxin and abscisic acid hormones. The expressions of OsSATs under different salt treatments conducted within study showed that OsSAT1;1, OsSAT1;2 and OSSAT1;3 were generally upregulated depending on different salt exposure times. However, OsSAT1;2 showed the highest expression levels when exposed to 3,12, and 24-hour salt treatments. Conversely, OsSAT2;2, OsSAT3 and particularly OsSAT2;1 were downregulated depending on salt exposure times. Watanabe et al. [45] stated that three cytosolic isoforms, SAT1;1, SAT3;1, and SAT3;2 genes in Arabidopsis contribute to 
seed development and SAT gene family plays essential roles for plant survive. Also, SAT3;1 and SAT3;2 isoforms of Arabidopsis play roles in plant development. In Arabidopsis, AtSAT2 and AtSAT4 are 10-100 times less transcribed compared to the major expressed SAT isoenzymes such as AtSAT1, 3 and $5[9,47]$. Everything considered, the varied expression patterns of OsSATs under various conditions show that they are dynamically regulated and OsSAT1;1 is the only OsSAT induced by various situmuli.

The expressional similarities among OsSATs may be originated from preserved residues in their active sites taking effect on their functions. As stated earlier, Asp (D), His (H), Gly (G), Thr (T), Arg (R), Ala (A), and Leu (L) are the conserved residues in active sites of OsSATs. His169, Asp154, His18, Arg203 and His204, Lys230, Arg253 in soybean were identified as active residues involved in reactions such as catalysis, oxyanion reaction intermediate, serine binding, CoA binding according to crystal structures and analysis of site-directed mutation data [48]. In this study, similar residues were identified in predicted active sites of OsSAT proteins.

\section{Conclusion}

A total of six OSSAT genes were identified in rice genome and variations at gene and protein structures were identified using bioinformatics approaches. It is found that jasmonic acid induced the expressions of OSSAT1;1, OSSAT2;1, and OSSAT2;2 whereas auxin and abscisic acid induced only OsSAT1;1. On the other hand, OSSAT1;1, OsSAT1;2 and OSSAT1;3 genes were upregulated under different exposure times of salt stress. OsSAT1;1 is the only OsSAT gene induced by various situmuli.

SAT gene family play specific roles in plant metabolism, particularly sulfur assimilation pathway depending on purifying selection which decreased genetic diversity of them. In addition to purifying selection, segmental and tandem duplications may lead to OsSATs to have more specific and selective roles in metabolic pathways which may have an effect on the plant's responses to abiotic and biotic stress conditions. Therefore, the findings can be used by plant breeders and genetic engineers to develop new rice varieties having optimal growth and stress tolerance.

\section{Declarations}

\section{Author contribution}

E.F and F.K. designed the study. E.F., F.K. and A.A. conducted the experiments and analyzed the data. All authors contributed to writing and editing the manuscript.

\section{Conflict of interest}

The authors declare that they have no conflict of interest

\section{Ethical approval}

This article does not contain any studies with human participants or animals performed by any of the authors

\section{References}

1. Leustek T (2002) Sulfate Metabolism. Arab B 1:e0017. https://doi.org/10.1199/tab.0017

2. Droux M (2003) Plant serine acetyltransferase: new insights for regulation of sulphur metabolism in plant cells. Plant Physiol Biochem 41:619-627. https://doi.org/10.1016/s0981-9428(03)00083-4

3. Haas FH, Heeg C, Queiroz R, et al (2008) Mitochondrial Serine Acetyltransferase Functions as a Pacemaker of Cysteine Synthesis in Plant Cells. Plant Physiol 148:1055-1067. https://doi.org/10.1104/pp.108.125237

4. Droux M, Ruffet M-L, Douce R, Job D (1998) Interactions between serine acetyltransferase and O-acetylserine (thiol) lyase in higher plants . Structural and kinetic properties of the free and bound enzymes. Eur J Biochem 255:235-245. https://doi.org/10.1046/j.1432-1327.1998.2550235.x

5. Wirtz M, Hell R (2006) Functional analysis of the cysteine synthase protein complex from plants: Structural, biochemical and regulatory properties. J Plant Physiol 163:273-286. https://doi.org/10.1016/j.jplph.2005.11.013

6. Romero LC, Aroca MÁ, Laureano-Marín AM, et al (2014) Cysteine and Cysteine-Related Signaling Pathways in Arabidopsis thaliana. Mol Plant 7:264276. https://doi.org/10.1093/mp/sst168

7. Kopriva S, Patron NJ, Keeling P, Leustek T (2008) Phylogenetic Analysis of Sulfate Assimilation and Cysteine Biosynthesis in Phototrophic Organisms. Sulfur Metab. Phototrophic Org. 31-58

8. Hell R, Jost R, Berkowitz O, Wirtz M (2002) Molecular and biochemical analysis of the enzymes of cysteine biosynthesis in the plant Arabidopsis thaliana. Amino Acids 22:245-257. https://doi.org/10.1007/s007260200012

9. Kawashima CG, Berkowitz O, Hell R, et al (2005) Characterization and Expression Analysis of a Serine Acetyltransferase Gene Family Involved in a Key Step of the Sulfur Assimilation Pathway in Arabidopsis. Plant Physiol 137:220-230. https://doi.org/10.1104/pp.104.045377 
10. Xiang X, Wu Y, Planta J, et al (2018) Overexpression of serine acetyltransferase in maize leaves increases seed-specific methionine-rich zeins. Plant Biotechnol J 16:1057-1067. https://doi.org/10.1111/pbi.12851

11. Goodstein DM, Shu S, Howson R, et al (2012) Phytozome: a comparative platform for green plant genomics. Nucleic Acids Res 40:D1178-D1186. https://doi.org/10.1093/nar/gkr944

12. Finn RD, Coggill P, Eberhardt RY, et al (2015) The Pfam protein families database: towards a more sustainable future. Nucleic Acids Res 44:D279D285. https://doi.org/10.1093/nar/gkv1344

13. Gasteiger E, Hoogland C, Gattiker A, et al (2005) Protein Identification and Analysis Tools on the ExPASy Server. Proteomics Protoc. Handb. 571-607

14. Hall TA (1999) Hall(1999)Nucleic_Acids_Symp_Ser_41,95.[BioEdit].pdf. Nucleic Acids Symp Ser 41:95-98. https://doi.org/citeulike-article-id:691774

15. Törönen P, Medlar A, Holm L (2018) PANNZER2: a rapid functional annotation web server. Nucleic Acids Res 46:W84-W88. https://doi.org/10.1093/nar/gky350

16. Bailey TL, Johnson J, Grant CE, Noble WS (2015) The MEME Suite. Nucleic Acids Res 43:W39-W49. https://doi.org/10.1093/nar/gkv416

17. Kimura M (1980) A simple method for estimating evolutionary rates of base substitutions through comparative studies of nucleotide sequences. $J$ Mol Evol 16:111-120. https://doi.org/10.1007/bf01731581

18. Rozas J, Ferrer-Mata A, Sánchez-DelBarrio JC, et al (2017) DnaSP 6: DNA Sequence Polymorphism Analysis of Large Data Sets. Mol Biol Evol 34:3299-3302. https://doi.org/10.1093/molbev/msx248

19. Jones DT, Taylor WR, Thornton JM (1992) The rapid generation of mutation data matrices from protein sequences. Bioinformatics 8:275-282. https://doi.org/10.1093/bioinformatics/8.3.275

20. Kumar S, Stecher G, Tamura K (2016) MEGA7: Molecular Evolutionary Genetics Analysis Version 7.0 for Bigger Datasets. Mol Biol Evol 33:1870-1874. https://doi.org/10.1093/molbev/msw054

21. Tajima F, Misawa K, Innan H (1998) The amount and pattern of DNA polymorphism under the neutral mutation hypothesis. Mutat. Evol. 103-107

22. Yang S, Zhang X, Yue J-X, et al (2008) Recent duplications dominate NBS-encoding gene expansion in two woody species. Mol Genet Genomics 280:187-198. https://doi.org/10.1007/s00438-008-0355-0

23. Darzentas N (2010) Circoletto: visualizing sequence similarity with Circos. Bioinformatics 26:2620-2621. https://doi.org/10.1093/bioinformatics/btq484

24. Lee T-H, Tang H, Wang X, Paterson AH (2012) PGDD: a database of gene and genome duplication in plants. Nucleic Acids Res 41:D1152-D1158. https://doi.org/10.1093/nar/gks1104

25. Sato Y, Takehisa H, Kamatsuki K, et al (2013) RiceXPro Version 3.0: expanding the informatics resource for rice transcriptome. Nucleic Acids Res 41:D1206-D1213. https://doi.org/10.1093/nar/gks1125

26. Sato Y, Namiki N, Takehisa H, et al (2013) RiceFREND: a platform for retrieving coexpressed gene networks in rice. Nucleic Acids Res 41:D1214D1221. https://doi.org/10.1093/nar/gks1122

27. Geourjon C, Deléage G (1995) SOPMA: significant improvements in protein secondary structure prediction by consensus prediction from multiple alignments. Bioinformatics 11:681-684. https://doi.org/10.1093/bioinformatics/11.6.681

28. Kelley LA, Sternberg MJE (2009) Protein structure prediction on the Web: a case study using the Phyre server. Nat Protoc 4:363-371. https://doi.org/10.1038/nprot.2009.2

29. Guex N, Peitsch MC, Schwede T (2009) Automated comparative protein structure modeling with SWISS-MODEL and Swiss-PdbViewer: A historical perspective. Electrophoresis 30:S162-S173. https://doi.org/10.1002/elps.200900140

30. Nguyen MN, Tan KP, Madhusudhan MS (2011) CLICK - Topology-independent comparison of biomolecular 3D structures. Nucleic Acids Res 39:24-28. https://doi.org/10.1093/nar/gkr393

31. Jones $\mathrm{P}$, Binns D, Chang H-Y, et al (2014) InterProScan 5: genome-scale protein function classification. Bioinformatics 30:1236-1240. https://doi.org/10.1093/bioinformatics/btu031

32. Szklarczyk D, Franceschini A, Wyder S, et al (2014) STRING v10: protein-protein interaction networks, integrated over the tree of life. Nucleic Acids Res 43:D447-D452. https://doi.org/10.1093/nar/gku1003

33. Kim JH, Lim SD, Jang CS (2020) Oryza sativa drought-, heat-, and salt-induced RING finger protein 1 (OsDHSRP1) negatively regulates abiotic stressresponsive gene expression. Plant Mol Biol 103:235-252. https://doi.org/10.1007/s11103-020-00989-x

34. Rademacher EH, Offringa R (2012) Evolutionary Adaptations of Plant AGC Kinases: From Light Signaling to Cell Polarity Regulation. Front Plant Sci 3:. https://doi.org/10.3389/fpls.2012.00250

35. Xu C, Luo F, Hochholdinger F (2016) LOB Domain Proteins: Beyond Lateral Organ Boundaries. Trends Plant Sci 21:159-167.

https://doi.org/10.1016/j.tplants.2015.10.010

36. Chen Y-C, Li C-L, Hsiao Y-Y, et al (2014) Structure and function of TatD exonuclease in DNA repair. Nucleic Acids Res 42:10776-10785. https://doi.org/10.1093/nar/gku732

37. Bækgaard L, Mikkelsen MD, Sørensen DM, et al (2010) A Combined Zinc/Cadmium Sensor and Zinc/Cadmium Export Regulator in a Heavy Metal Pump. J Biol Chem 285:31243-31252. https://doi.org/10.1074/jbc.m110.111260

38. Marks DS, Colwell LJ, Sheridan R, et al (2011) Protein 3D Structure Computed from Evolutionary Sequence Variation. PLoS One 6:e28766. https://doi.org/10.1371/journal.pone.0028766

Page $10 / 17$ 
39. Sankararaman S, Sha F, Kirsch JF, et al (2010) Active site prediction using evolutionary and structural information. Bioinformatics 26:617-624. https://doi.org/10.1093/bioinformatics/btq008

40. Cvijović I, Good BH, Desai MM (2018) The Effect of Strong Purifying Selection on Genetic Diversity. Genetics 209:1235-1278. https://doi.org/10.1534/genetics.118.301058

41. Yang Z, Yoder AD (1999) Estimation of the Transition/Transversion Rate Bias and Species Sampling. J Mol Evol 48:274-283. https://doi.org/10.1007/pl00006470

42. Šmarda P, Bureš P, Horová L, et al (2014) Ecological and evolutionary significance of genomic GC content diversity in monocots. Proc Natl Acad Sci 111:E4096-E4102. https://doi.org/10.1073/pnas.1321152111

43. Panchy N, Lehti-Shiu M, Shiu SH (2016) Evolution of gene duplication in plants. Plant Physiol 171:2294-2316. https://doi.org/10.1104/pp.16.00523

44. Kuo Y-T, Chao Y-T, Chen W-C, et al (2019) Segmental and tandem chromosome duplications led to divergent evolution of the chalcone synthase gene family in Phalaenopsis orchids. Ann Bot 123:69-77. https://doi.org/10.1093/aob/mcy136

45. Watanabe M, Mochida K, Kato T, et al (2008) Comparative Genomics and Reverse Genetics Analysis Reveal Indispensable Functions of the Serine Acetyltransferase Gene Family in Arabidopsis. Plant Cell 20:2484-2496. https://doi.org/10.1105/tpc.108.060335

46. Ben-Hur A, Brutlag D (2006) Sequence Motifs: Highly Predictive Features of Protein Function. Featur. Extr. 625-645

47. Wirtz M, Droux M (2005) Synthesis of the sulfur amino acids: cysteine and methionine. Photosynth Res 86:345-362. https://doi.org/10.1007/s11120005-8810-9

48. Yi H, Dey S, Kumaran S, et al (2013) Structure of Soybean Serine Acetyltransferase and Formation of the Cysteine Regulatory Complex as a Molecular Chaperone. J Biol Chem 288:36463-36472. https://doi.org/10.1074/jbc.m113.527143

\section{Figures}

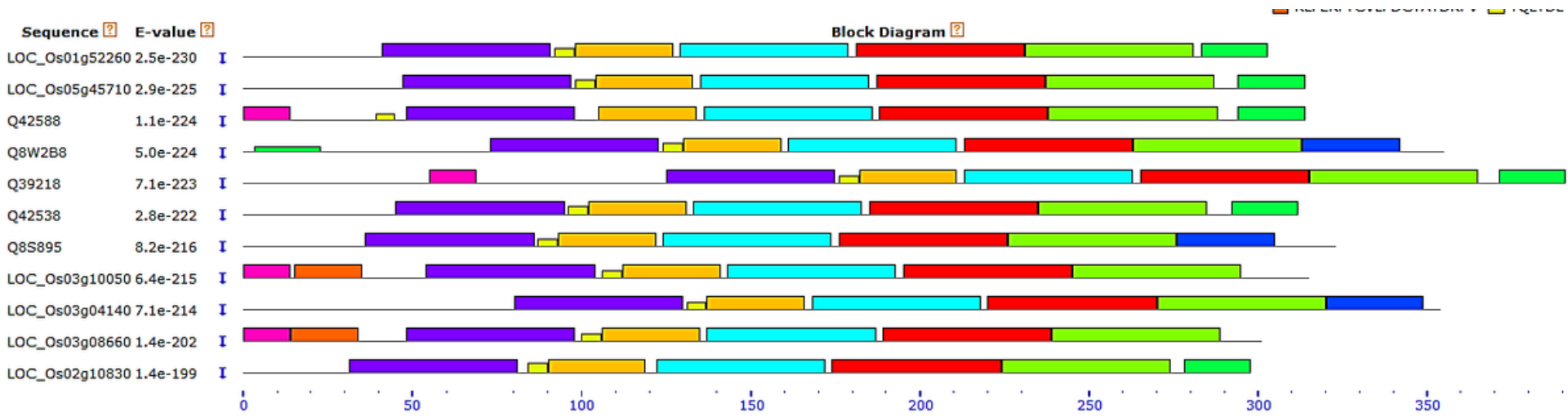

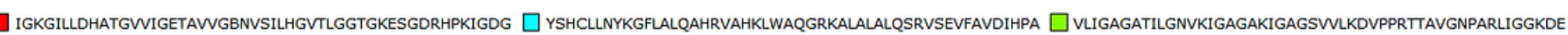

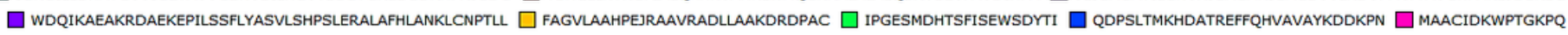

\section{Figure 1}

Block diagram of conserved motifs in 11 SAT protein sequences from rice and Arabidopsis using MEME server. Each color represents the distinct motif structure. 


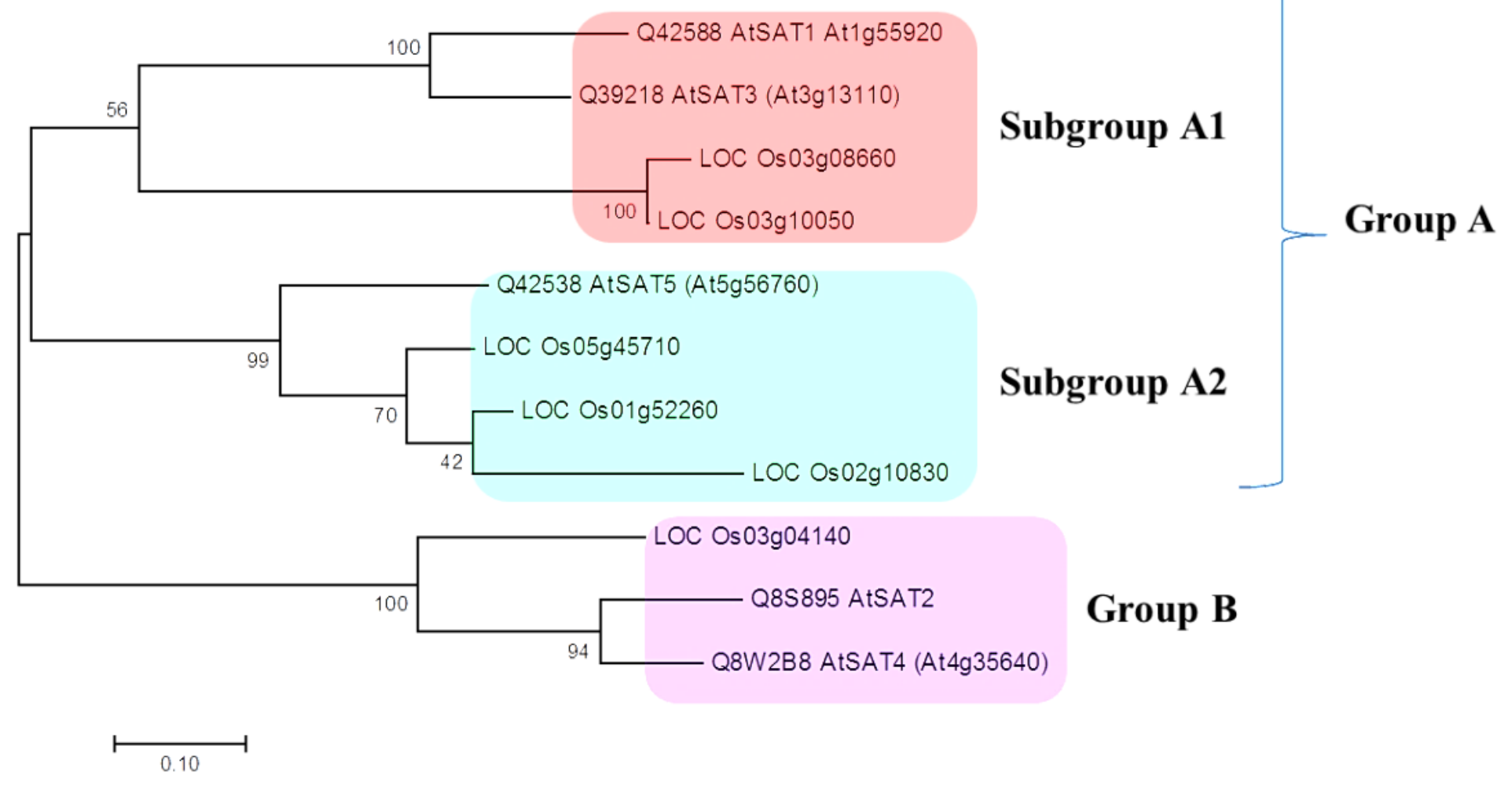

Figure 2

Phylogenetic tree of SAT protein family based on SAT amino acid sequences of rice and Arabidopsis. 

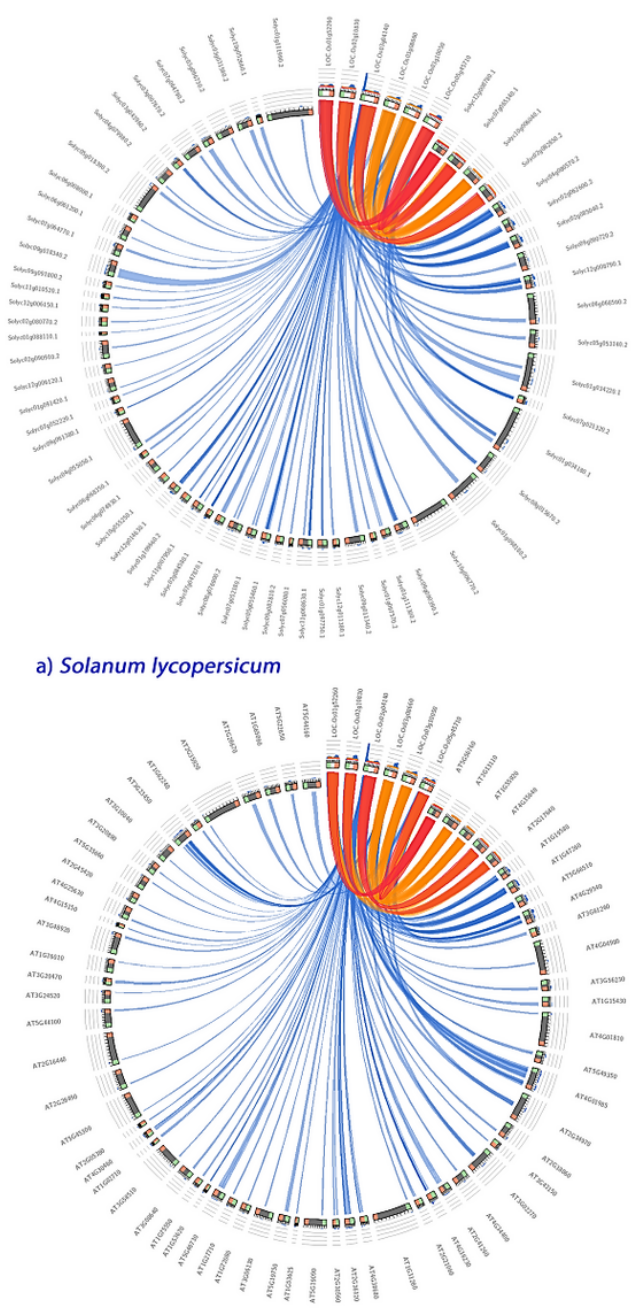

c) Arabidopsis thaliana
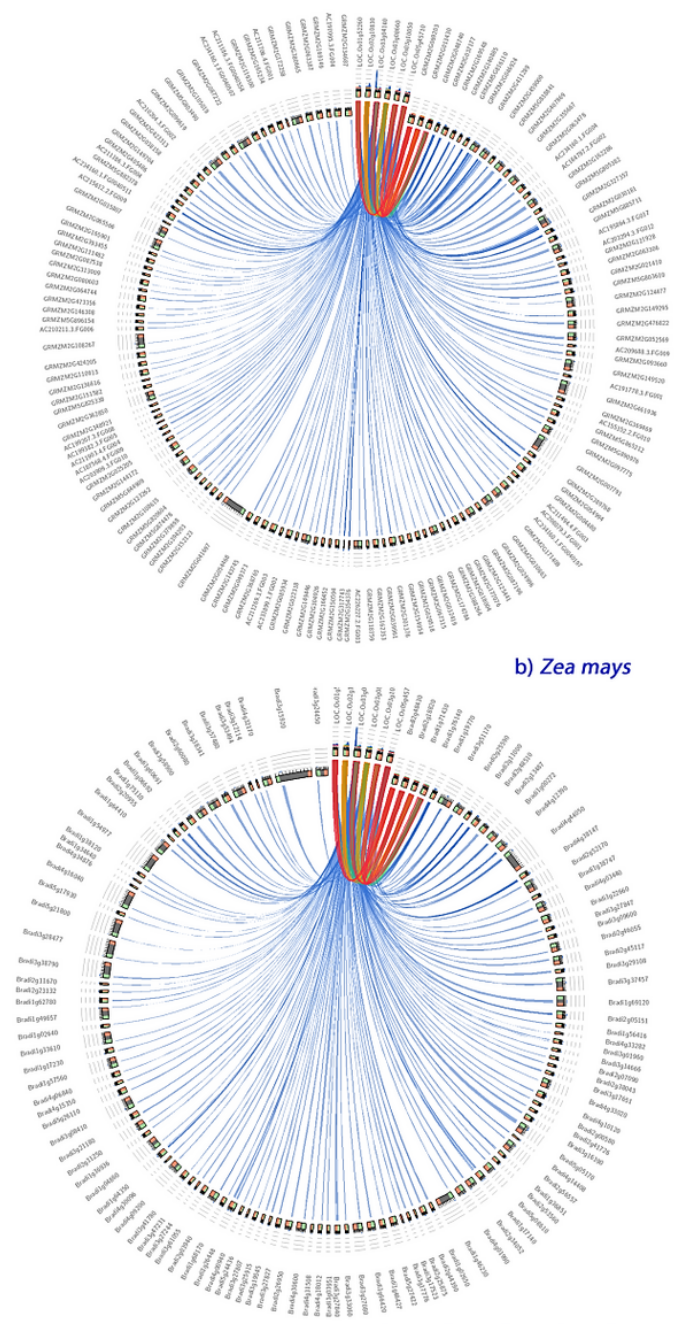

d) Brachypodium distachyon

\section{Figure 3}

Synteny analyses of OsSAT genes in tomato (a), maize (b), Arabidopsis (c), and B. distachyon (d). Blue lines in the background indicate the collinear blocks within rice and other plant genomes whilst the red and yellow lines show the syntenic SAT gene pairs. 


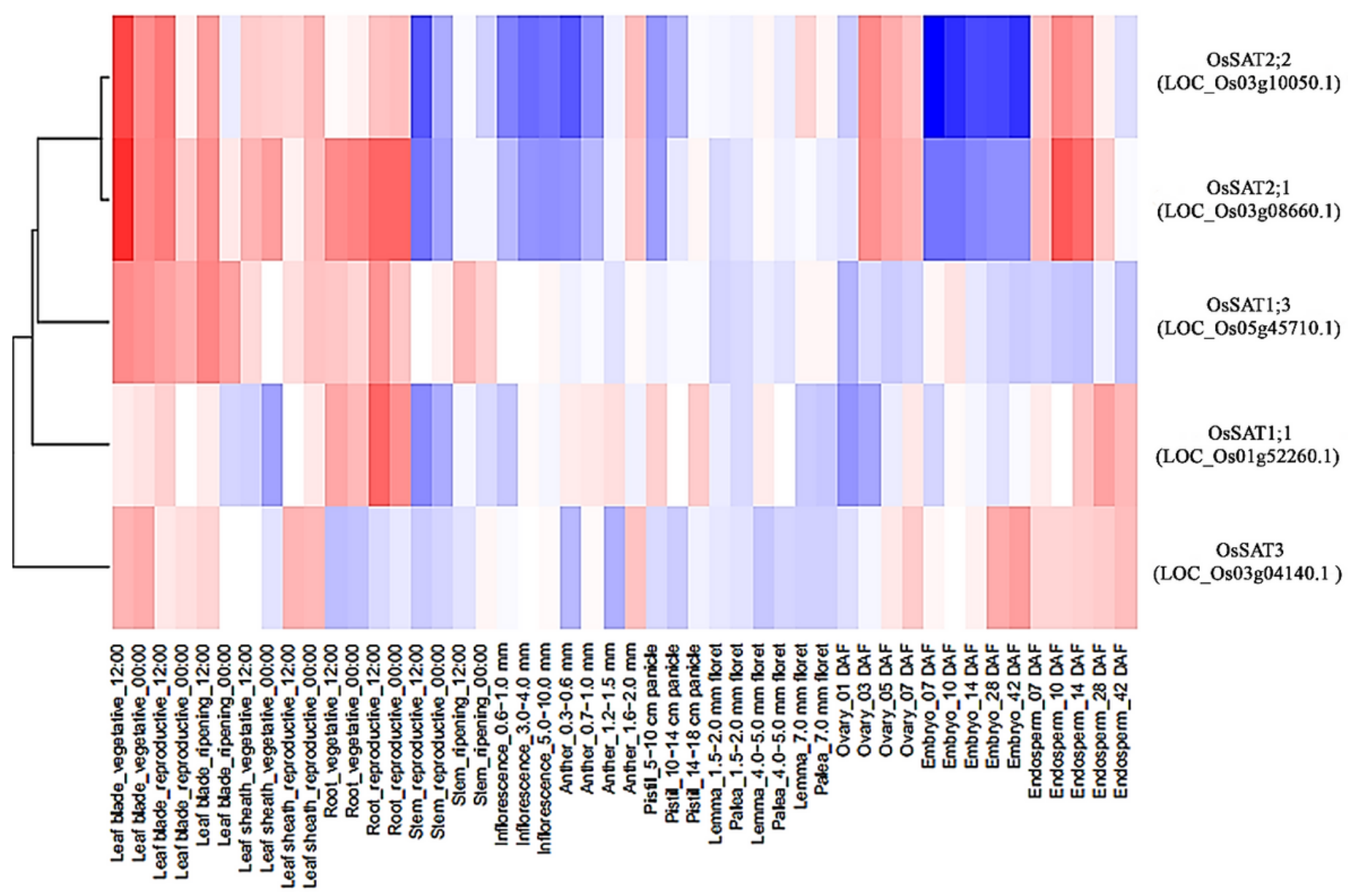

Figure 4

Spatio-temporal OsSAT gene expression of various tissues/organs throughout entire growth in the field. The expression data is retrieved from Rice Expression Profile Database (RiceXPro) 


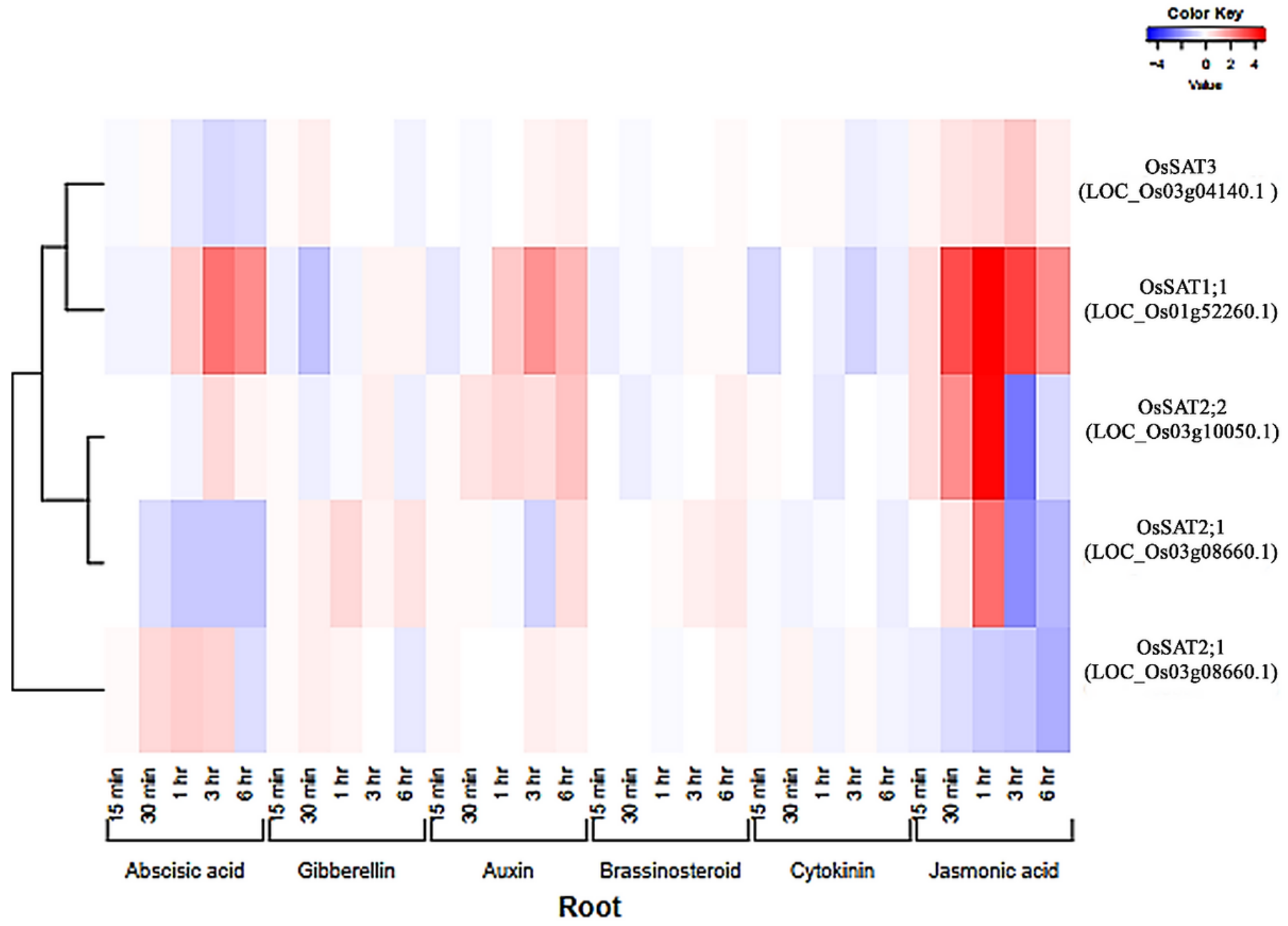

Figure 5

Global OsSAT gene expression profile in response to plant hormones. The expression data is retrieved from Rice Expression Profile Database (RiceXPro) 


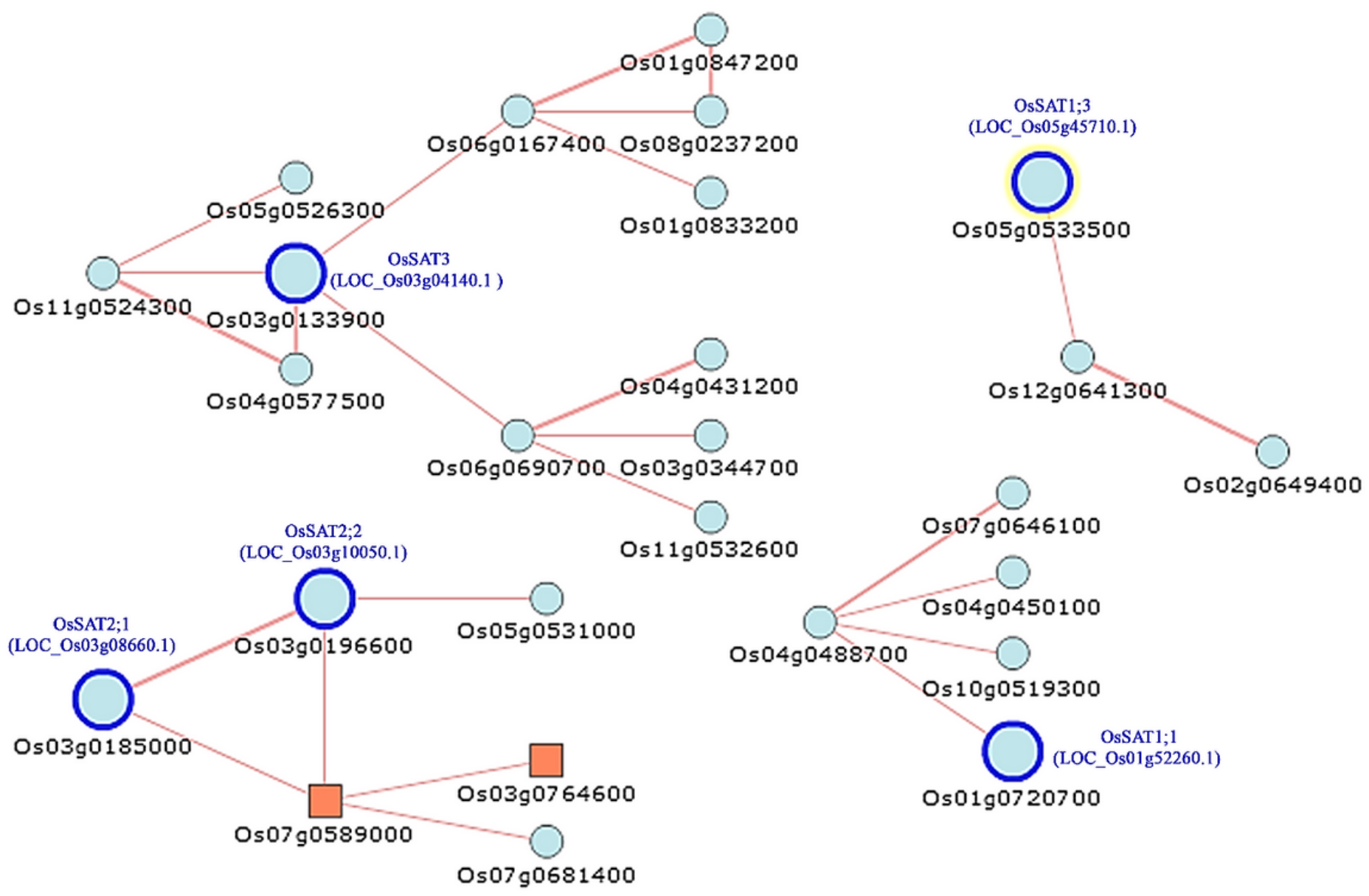

Figure 6

Co-expression networks of OsSAT genes (blue-circled). The each network was generated using the data on RiceFREND gene co-expression database.

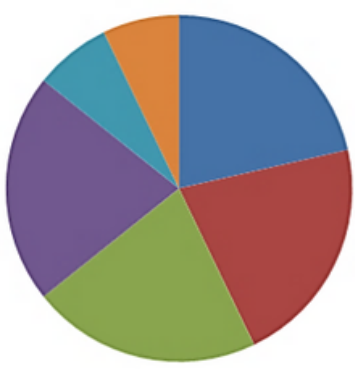

A

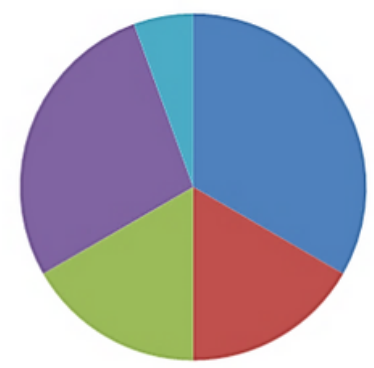

B

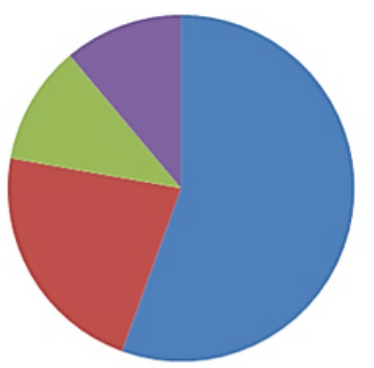

C

| G0:0005737 cytoplasm acetyltransferase activity

= 60:0010333 terpene synthase activity

a $\mathrm{GO}: 0000287$ magnesium ion binding

= GO:0008270 zinc ion binding

= 60:0005515 prote in

- 60:0008652 cellular am ino acid biosynthetic component of membrane II GO:0044424 intracellular part In 60:0043227 membrane. bounded organe lle

\section{Figure 7}

GO annotation of OsSAT proteins using PANNZER server. The annotation was done on three categories: (a) biological process, (b) molecular function, and (c) cellular component. 


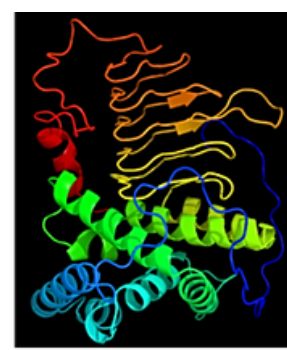

A- AtSAT1

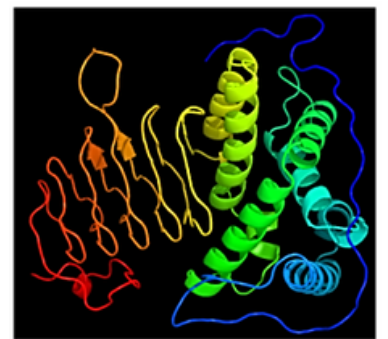

F- LOC_Os03g08660

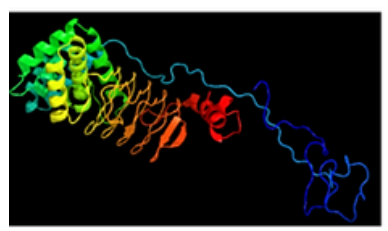

K- LOC_Os03g04140

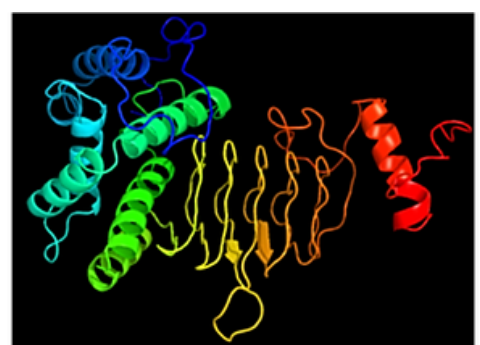

B- AtSAT2

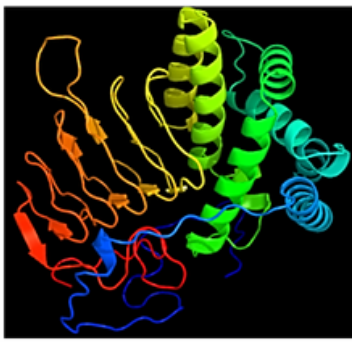

G- LOC_Os03g10050

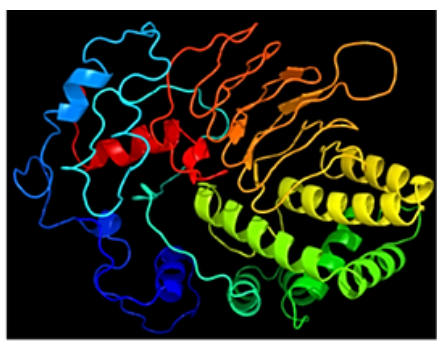

C- AtSAT3

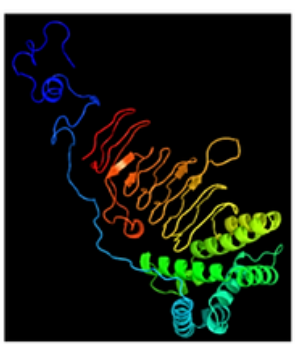

D- AtSAT4

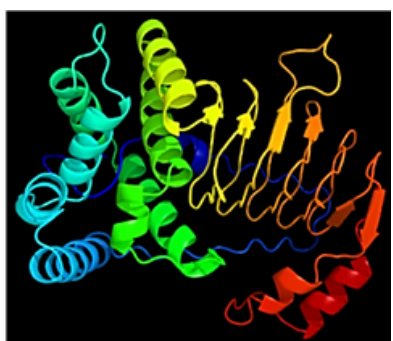

E- AtSAT5

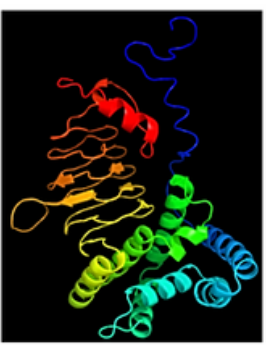

H- LOC_Os01g52260

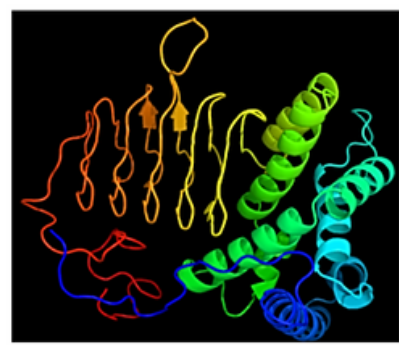

I- LOC_Os02g10830

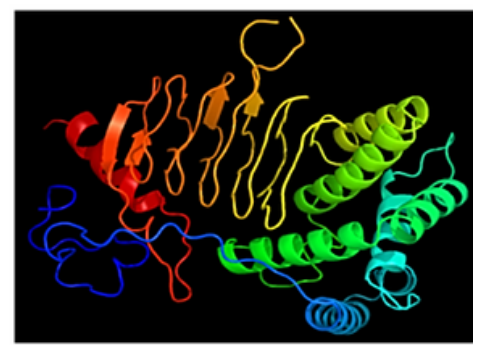

J- LOC_Os05g45710

Figure 8

The predicted 3D model of Arabidopsis and rice SAT proteins
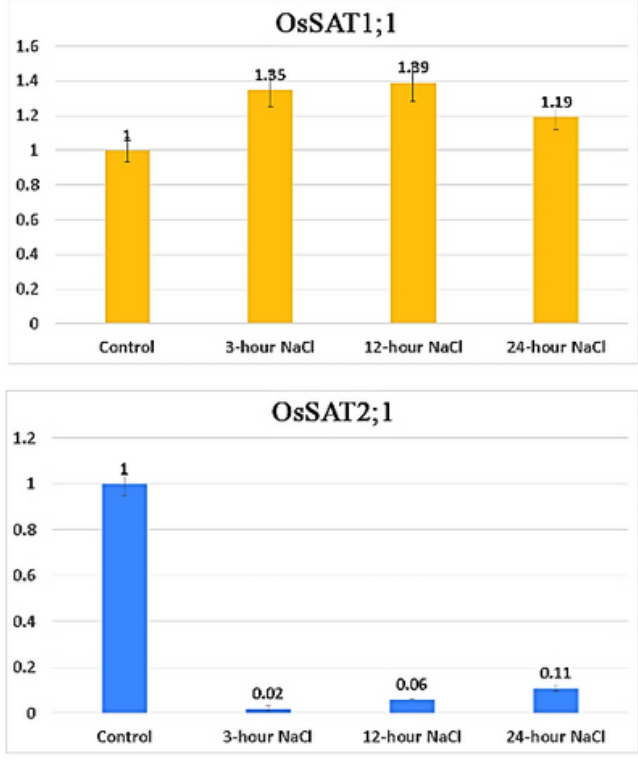

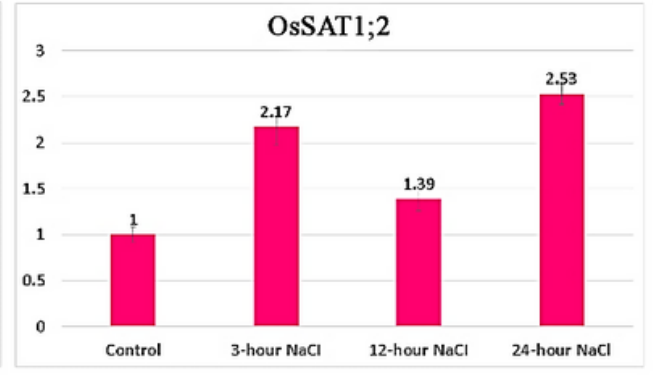

OsSAT2;2

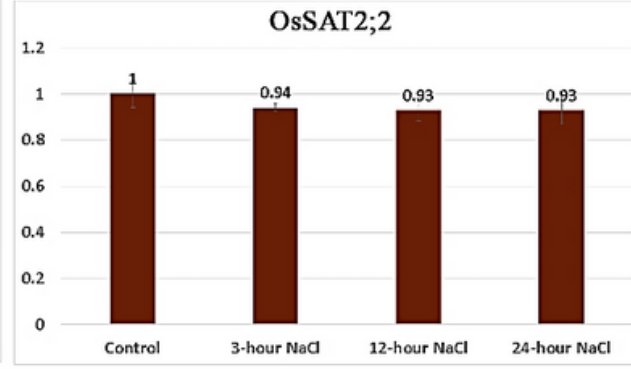

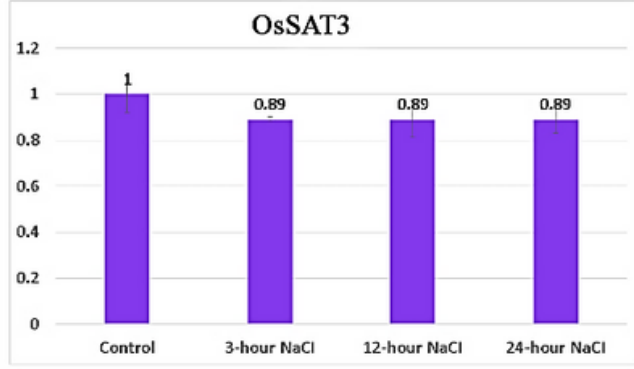

OsSAT1;3

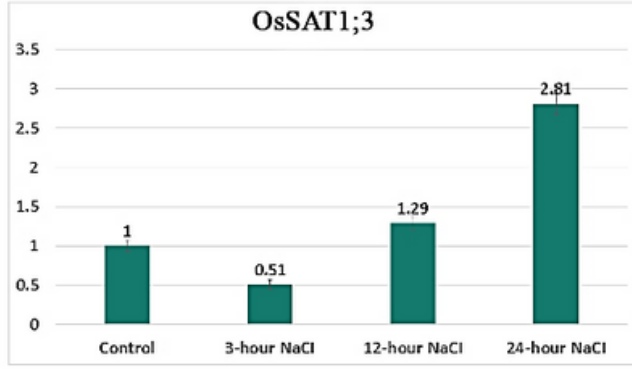

Figure 9

The expression of OsSATs under salt stress. Each gene was shown in different color. The error bars on the graphs show the standard deviations of expressions. 